\title{
An Analytical Study of LEACH Routing Protocol for Wireless Sensor Network
}

\author{
Mohd Nazim Jambli, Mohd Imran Bandan, Kosheila Sundram Pillay \\ Faculty of Computer Science and Information Technology \\ Universiti Malaysia Sarawak, Kota Samarahan \\ 94300 Sarawak, Malaysia \\ \{jmnazim,bmimran\}@unimas.my, kosheilapillay@gmail.com
}

\author{
Sinarwati Mohamad Suhaili \\ Centre for Pre-University Studies \\ Universiti Malaysia Sarawak, Kota Samarahan \\ 94300 Sarawak, Malaysia \\ mssinarwati@ preuni.unimas.my
}

\begin{abstract}
The use of low power sensor nodes to collect useful sensing information effectively is critical for any wireless sensor network (WSN) application to last longer. To increase network lifetime, energy consumption is considered as one of an essential performance metric. Most of the current proposed routing protocols proposed to reduce the amount of energy consumed and to increase the network lifetime. The common pioneer hierarchical routing protocol for wsn such as Low Energy Adaptive Cluster Hierarchical Routing (LEACH) is also proposed to improve the energy efficiency of WSN. LEACH is a cluster based routing protocol in which sensor nodes are combined together to form separate clusters and every cluster has a leader node named cluster head $(\mathrm{CH})$. In this paper, we have done the analytical study of LEACH protocol to identify to what extent LEACH protocol can perform in terms of average energy consumption and packet loss for different data rate.
\end{abstract}

Index Terms-Wireless Sensor Network; LEACH routing protocol; energy consumption; NS-2

\section{INTRODUCTION}

Communication networks based on sensors have begun to appear as a potential platform to monitor the physical environments [1] and for control applications because of the growing technological developments in power-saving electronics, sensors, and wireless communications [2]. A wireless sensor network (WSN) is one such network that is equipped with sensors communicating data packets to a predefined recipient base station (BS) [3].

WSN consists of a group of static sensor nodes with reduced processing capacities and power supply to transmit data over radio links [2], [4]. Sensors are usually deployed in specific areas to sense and communicate different environmental conditions such as temperature, pressure, sound, humidity, etc [5], [6]; often-times, in hazardous and hostile areas [1], [6]. Sensor nodes are fundamentally battery devices; therefore, power resource has been a constant issue in the reduction of lifetime of the wireless sensor networks [3], [7], [8]. Hence, energy efficiency plays a role in the extension of the WSN lifetime [8]. As a result, many routing protocols have been proposed to improve the energy efficiency and lifetime of wireless sensor networks [5].

\section{LEACH ROUTING PROTOCOL}

Routing protocols in wireless sensor networks can be categorized into four different classes, namely network structure, topology-based, communication model, and reliable routing classes [9]. Under the network structure, routing protocols can be further classified into flat routing, location or geographic based routing and hierarchical routing protocols [10]. In a flat routing network, all sensor nodes have equal roles and are less scalable [11]. Hierarchical or cluster-based routing protocols, on the other hand, offer better energy efficiency rates and scalability because of its structural organization [10], [11].

In a hierarchical network, the network is divided into a number of clusters and some sensor nodes have a special role than the rest, which are known as the cluster heads (CHs) [10], [11]. Low energy adaptive clustering hierarchy (LEACH) [12] protocol is an example of hierarchical routing protocol and is the first popular routing protocol found to be the most efficient against the conventional routing protocols [6]. In LEACH, the network is divided into different clusters, each with its own cluster head selected during each round of data transmission [13]. CHs collect, aggregate and transmits data packets received from other neighbouring nodes to the BS [11]. The operation of LEACH routing protocol is comprised of two phases, which are the set-up phase and the steady state phase [14], [15]. In the set-up phase, processes such as the $\mathrm{CH}$ selection, cluster formation, and TDMA scheduling are executed. During the $\mathrm{CH}$ selection, each node participates in the process by generating a random value between 0 and 1 , and if the generated value is less than the threshold value, then that node becomes the $\mathrm{CH}$ [11]. In the steady state phase, the sensed data packets from regular nodes are communicated to the $\mathrm{CH}$ and from $\mathrm{CH}$ to $\mathrm{BS}$ using the TDMA schedule [2], [11].

In this paper, the performance of the LEACH routing protocol under different simulation experiments will be evaluated in order to identify to what extent LEACH protocol can perform in terms of average energy consumption and packet loss for different data rate. The rest of the paper is organised follows. Section III describes the performance evaluation of LEACH routing protocol in terms of the simulation tool, simulation set-up, evaluation metrics, and the experiments. Section IV presents the simulation analysis of the routing protocol based on the different experiments and discusses the simulation results. Section V concludes the results obtained and suggests some future works in the field. 